\title{
Enhancement of Fundus Images for Diagnosing Diabetic Retinopathy using B-Spline
}

\author{
Tayba Bashir ${ }^{1}$, Khurshid Asghar ${ }^{2}$, Mubbashar Saddique ${ }^{3}$, Shafiq Hussain ${ }^{4}$, Inam Ul Haq ${ }^{5}$ \\ Department of Computer Science, University of Sahiwal, Sahiwal, 57000, Pakistan ${ }^{1,4}$ \\ Department of Computer Science, University of Okara, Okara, 56300, Pakistan 2,3,5
}

\begin{abstract}
Medical images, such as CT scan, MRI, X-ray, mammography and fundus are commonly used in medical diagnosis process and helpful to improve diagnose of disease in a better way and reduces the chances of ambiguous perceptions. Medical images are mostly available in low contrast, brightness and noisy form due to camera/ radio waves intrinsic properties while capturing, which disrupts the diagnosis process using medical images. Enhancement of these images can improve the diagnosis process. The proposed enhancement technique of fundus images is based on the B-spline interpolation, in which intensity transformation curve is based on the control points of the curve. Messidore and Drive datasets of Diabetic Retinopathy (DR) are used to evaluate the proposed enhancement technique. Results shows that the fundus images have reasonable visual and quantitative enhancement when performed comparison with recent techniques. Results are of evidence that the proposed approach has substantial outcome and preserves important information of fundus images by lowering noise.
\end{abstract}

Keywords-B-spline; medical images enhancement; fundus images; diabetic retinopathy; interpolation

\section{INTRODUCTION}

Contrast enhancement of images is a wide area in digital image processing. The contrast in images make it better to visually analyze and understand the shape of the object clearly. The contrast of images depends on environmental effects like weak lenses of capturing device, low light due to fog and clouds, unprofessional photographer. The images captured under these circumstances have contrast alteration, high noise, and color vanishing [1].

Intensity enhancement makes the objects and background separate to attain the hidden detail [2]. Digital images have many applications in every field of life like medical, security, forensic, education, satellites, recognition, and pattern detection. Contrast enhancement techniques have two types, direct enhancement in which we use fuzzy logic, adaptive neighborhood contrast enhancement and segmentation. While the indirect contrast enhancement is based on histogram changes of image like histogram equalization, contrast limited adaptive histogram equalization and recursive mean separate histogram equalization [3]. Digital image processing is popular for two areas. One is the images information for human use and diagnosis. This is helpful to treat a patient with early diagnosis. The second part is based on processing, storing, altering for machine learning like bio-sensing and identifying.
Medical images enhancement is one the biggest need of the human body tissues to diagnose, monitoring and interpretation of diseases. Most of the medical images are in gray scan like CT scan, X-ray and MRI. The X-rays are frequently used for image elevated compactness like bone. The Computed tomography and Magnetic Resonance imaging used for low density tissues [4]. Enhancement in the medical field is useful to identify diseases like tumors, liver distortion, heart breakage, bone fracture, muscular pain and tissues pull etc. In medical images, a general problem is to capture images in motion form for real time assessment. The blur and deformed images are hard to understand and assessed correctly. The edge detection and organ identification are the most common part of preprocessing technique. There are so many techniques that help to enhance edges and organs in medical images. Medical images have darkness in objects and borders of these objects have noise, which is a basic factor to create discontinuities in images [5]. We have multiple techniques to enhance intensity of medical images like Image Negative, Power Law, Logarithmic Transformation, Histogram Equalization (HE), Bezier Curve for Contrast Enhancement (BCCE), Bezier curve using Fourier Transformation etc. There are many techniques that are studied to enhance image quality and accuracy without loss of information. To increase the low intensity of image the geometric Multiscale differential operators' technique was introduced [6] and it helped to improve edges and corners with returning the best mammographic result.

The medical image enhancement is basically depending on the Intensity transformation. The Breast MRI result observed using the contrast enhancements using fuzzy type1 and fuzzy type 2 provides brightness of image. The colorful medical images are needed to enhance with better pixel results. The interactive color image segmentation is performed with the combination of Bezier curve, which give a balanced intensity of images. Medical images have many side effects one of the majors is radiation which is harmful for the human body. Hence, image enhancement enhances blur image and reduces noise of thermal images. Thus, breast cancer images are enhanced by applying reducing radiation and advanced electric equipment [7]. Discrete wavelet transformation is used for image compression, de-noising, segmentation etc. The discrete wavelet provides great results with coefficient domain [8]. The cubic spline shows better results with thresholding method and curve fitting technique. The piecewise polynomial is converted into small segments and enhances image [9]. 
The proposed technique is B-spline based intensity transformation of dark images. The results generated by proposed method of image enhancement are compared both visually and quantitatively using entropy, peak signal to noise ratio, contrast to noise ratio and structure similarity index image enhancement evaluation measures [2].

\section{A. Diabetic Retinopathy (DR)}

Diabetes has many serious obstacles but diabetic retinopathy such severe impediment which requires consideration at a very beginning level [10]. The diabetic retinopathy is increasing eye disease that intensifies with time source of permanent harm to retina and eventually turns into sightlessness. The recent studies show that $75 \%$ of patients have elevated glucose levels for twenty years are identified with diabetic retinopathy. Blindness is a major cause in the United States of America suffers adults due to diabetics [11]. The blindness risk in diabetic patients is $20 \%$ more than normal persons. The identification of retinopathy in patients is a big issue these days. India also suffers to deal with this problem as it is on sixth number in world countries who have diabetic patients [12]. It is the need of time to improve the diagnostic accuracy in ophthalmology. It is difficult to identify the retinoic mellitus by small observation. It is a basic step to identify the internal blood leakage, d-shape of small veins of eye for a doctor. If caught at an early stage a patient is recovered early or may be treated with a lens operation. Identification of diabetic retinopathy at an early stage of disease rescues it from loss of sight and reoperation. Most of the images are captured by oscilloscopes and it is not clear due to environmental effects, camera lens or may be the noise that affects images badly. Due to all these reasons, a new algorithm is trying to develop that enhances image without loss of data and returns great results to deal with retinopathy disease easily at an early stage. A comparison of state-of-theart image enhancement techniques is shown in Table I.

State-of-the art image enhancement techniques like histogram equalization (HE), adaptive histogram equalization (AHE), contrast limited adaptive histogram equalization (CLAHE), exposure-based sub image histogram equalization (ESIHE) in histogram family and others like interpolation, modulation, classification and segmentation are commonly used to enhance medical images. The outcomes are not satisfied for multiple images due to enhanced contrast with increasing noise. Some techniques are over enhanced contrast ratios due to modification of unnecessary areas; some enhance contrast at the rate of loss of image information. Similarly, enhancement of medical images is a difficult task with keeping all the information and removing noise with increasing contrast of necessary areas and edges of image. The focus of this research is to develop a robust method which enhances DR images.

Rest of the paper is organized as follows: Section II describes the proposed methodology. Implementation and results are presented in Section III. Section IV is about conclusion and future work.

\section{TABLE I. STATE OF THE ART COMPARISON}

\begin{tabular}{|c|c|c|c|}
\hline Reference & Approach & Pros & Cons \\
\hline Zhao et al. [4] & $\begin{array}{l}\text { Luminance and gradient } \\
\text { modulation }\end{array}$ & $\begin{array}{l}\text { Reduced dynamic range of luminance level using gradient } \\
\text { computation. Enhancement is done at local level instead of globally. }\end{array}$ & Not clear the image edges detail. \\
\hline Asghar et al. [2] & $\begin{array}{l}\text { Bezier Curve using } \\
\text { Fourier Transformation }\end{array}$ & $\begin{array}{l}\text { Automatic reorganization of the type of input image. } \\
\text { Enhanced images by modifying frequency domain. }\end{array}$ & $\begin{array}{l}\text { Bezier curve have statics } \\
\text { behavior, this may cause loss of } \\
\text { information. }\end{array}$ \\
\hline Akila et al. [3] & $\begin{array}{l}\text { Indirect contrast } \\
\text { enhancement }\end{array}$ & $\begin{array}{l}\text { Contrast enhancement using Histogram and its types. } \\
\text { Better results for low contrast images. }\end{array}$ & $\begin{array}{l}\text { Detail of images can't be viewed } \\
\text { clearly. }\end{array}$ \\
\hline Du-Yih Tsai et al. [8] & $\begin{array}{l}\text { Wavelet coefficient } \\
\text { mapping functions }\end{array}$ & $\begin{array}{l}\text { Convert image non-liner coefficient into discrete wavelet coefficient. } \\
\text { Using fast Fourier transformation for low resolution image } \\
\text { enhancement. }\end{array}$ & $\begin{array}{l}\text { Improvement in graph cut with } \\
\text { new edge weight. }\end{array}$ \\
\hline Yu-Ping et al. [6] & $\begin{array}{l}\text { Multiscale differential } \\
\text { operators. }\end{array}$ & $\begin{array}{l}\text { High frequency features like edges are characterized } \\
\text { Improved classification of chromosomes and qualitative contrasts. }\end{array}$ & $\begin{array}{l}\text { Increase memory requirement } \\
\text { due to completion of multiple } \\
\text { process. }\end{array}$ \\
\hline Thierry Blu et al. [13] & $\begin{array}{l}\text { Fractional spline } \\
\text { wavelet transformation }\end{array}$ & $\begin{array}{l}\text { Its return positivity and compact support } \\
\text { Whitened noise and enhanced low contrast area. }\end{array}$ & $\begin{array}{l}\text { It works with alpha elements } \\
\text { only. }\end{array}$ \\
\hline Umar Talha et al. [14] & $\begin{array}{l}\text { Hermit based } \\
\text { interpolation }\end{array}$ & $\begin{array}{l}\text { Under sampled reconstruction of image angel's help to reduce } \\
\text { radiations. } \\
\text { Filtered back projection returns visible artifacts in the output image and } \\
\text { has } 94 \% \text { accuracy. }\end{array}$ & $\begin{array}{l}\text { Spatial filtering-based post } \\
\text { processing is needed. }\end{array}$ \\
\hline Najid et al. [15] & $\begin{array}{l}\text { Classification and } \\
\text { segmentation }\end{array}$ & $\begin{array}{l}\text { Breast region segmented into small areas for exact diagnosis with } \\
\text { smooth interpolation. }\end{array}$ & $\begin{array}{l}\text { Loss of some information due to } \\
\text { inappropriate detail. }\end{array}$ \\
\hline Lehmann et al. [16] & $\begin{array}{l}\text { B-spline using Fourier } \\
\text { transformation }\end{array}$ & $\begin{array}{l}\text { Interpolation performs using down sampling to get improved results. } \\
\text { Extract luminance and represent naturalness. }\end{array}$ & $\begin{array}{l}\text { The dark images need to } \\
\text { reconstruct before } \\
\text { implementation. }\end{array}$ \\
\hline Xueyang et al. [17] & $\begin{array}{l}\text { Fusion methods to } \\
\text { enhance luminance }\end{array}$ & $\begin{array}{l}\text { Blended of multiple enhancement approaches return great results. It } \\
\text { can also use as post processing for any type enhancement. }\end{array}$ & $\begin{array}{l}\text { Haze removal is required for } \\
\text { better outcomes. }\end{array}$ \\
\hline
\end{tabular}




\section{Proposed Methodology}

To enhance medical images B-spline interpolation-based technique is proposed in this paper. It's based on three steps. In the first step the RGB are converted into $\mathrm{YCbCr}$ color space and extract ' $\mathrm{Y}$ ' channel. In the second step apply Bspline transformation on the extracted $\mathrm{Y}$ channel. In the third step merge the enhanced $\mathrm{Y}$ channel with $\mathrm{Cb}$ and $\mathrm{Cr}$ and get resulted enhanced output image.

\section{A. B-Spline}

The proposed image enhancement technique is based on B-spline curve which is used to enhance medical images dynamically with securing all information by intensity transformation using. The B-spline curve is based on control points; value of a curve is 0 to 255 . The $\mathrm{P} 1$ and $\mathrm{P} 2$ points are changed according to the distance $(D)$ is measured to enhance an image contract. B-spline is looking like Bezier curve accepts its dynamic nature as shown in Fig. 1.

The blending function use control points to make curve. The $n+1$ control points $P 0, P 1, \ldots, P n$ and a knot vector $T$, the B-spline curve of degree $p$ defined by these control points. Polynomial b-spline has similar properties with Bezier curve. The B-spline interpolation has interval of point $[a, b]$. The degree of curve is denoted as $p$, so curve $\leq p$. The knot vector of B-spline curve is denoted $T=\left\{t_{0}, \ldots, t_{m}\right\}$ with value of parameter taken as $t_{j} \leq t_{i}+1, i=0, \ldots, m-1$. Control points are taken as $b o \ldots b_{\text {n. }}$. B-spline basic function is defined as:

$P(u)=\sum_{i=0}^{n} p_{i} N_{i, D}(u)$

The number of control points in B-spline are determined by D-1. The basic function defines itself repeatedly. B-spline function conditions are shown in

$N_{i, 1}(u)=1 \Rightarrow t_{i} \leq u<t_{i+1}$

$=0$, otherwise

The polynomial function of $n^{\text {th }}$ degree is described by equation (3).

$N_{i, d}(u)=\frac{\left(u-t_{i}\right) N_{i, D-1}(u)}{t_{i+D-1}-t_{i}}, \frac{\left(t_{i+d}-u\right) N_{i+1, D-1}(u)}{t_{i+D,}-t_{i+1}}$

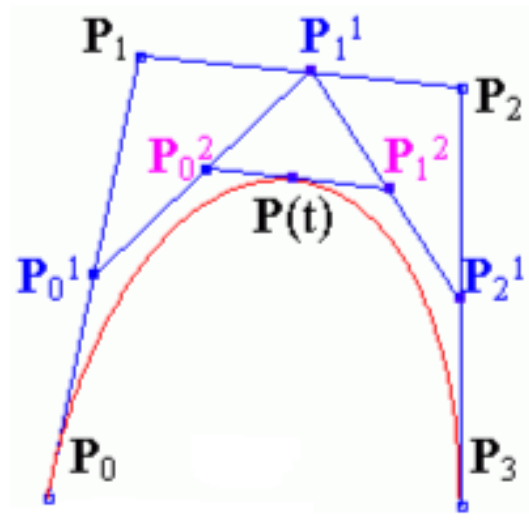

Fig. 1. B-Spline Curve.
The B-spline is similar to Bezier curve, but it has to pass out all the points known as interpolation; therefore, it yields better intensity transformation curve.

\section{B. Algorithm}

1) Select the input image

2) Covert to YCbCr color space

3) Calculate histogram of $Y$ channel of image to categorize the image as dark, bright, low contrast, high contrast and backlight.

4) Calculate movement distance $(D)$ using equation (4)

$D=\left(2^{(N-1)}\right) \times T c$, (4) where $N$ is the total number of pixels with frequencies greater than zero and $\alpha T_{\mathrm{c}}$ is calculated) with contrast ratio formula given in equation (2):

$T c=\frac{N}{(\operatorname{Lmax}-\operatorname{Lmin}+1)}$

where, Lmax and Lmin are pixels with maximum and minimum luminance values respectively.

5) Apply equation (1) and calculate new position of control points $P 1$ and $P 2$, according to the calculated movement distance $D$, the end points $P 0$ and $P 3$ remain fixed at $(0,0)$ and $(255,255)$, respectively. Calculation of middle control points for all possible types of images are illustrated in equations (6), (7) and (8), respectively.

a) for over dark images:

$P 1=P 0+\left(\frac{D}{2}, 0\right)$ and $P 2=P 3-\left(\frac{D}{2}, 0\right)$

b) for over bright and back light images:

$P 1=P 0+\left(\frac{D}{2}, 0\right)$ and $P 2=P 3-\left(\frac{D}{2}, D\right)$

c) for over low contrast images:

$P 1=P 0+\left(\frac{D}{2}, 0\right)$ and $P 2=P 3+(1,1)$

6) Perform intensity transformation using B-spline curve produced in step 5 on Y channel of the images to compute new histograms for mapping of new luminance values.

7) Finally, transformed $\mathrm{Y}$ is concatenated with $\mathrm{Cb}$ and $\mathrm{Cr}$ to get output image.

This procedure provides the enhanced fundus images without loss of information.

\section{Dataset Description}

The algorithm results tested on fundus images selected from two publicly available dataset i.e., DRIVE and MESSIDOR. The details are given under: Messidor is a fundus images dataset of 1200 lossless images. These images take at $45^{\circ}$ with FOV camera in different directions [18]. The other online available dataset named as Drive consists of 40 lossless images in colored format with clinical expert remarks [12]. These images are categories into two parts: training and testing. Image resolution is taken as $768 \times 584$ pixels with a field of view of $45^{\circ}$. The four DR images (see Fig. 2) are taken to show the results. 


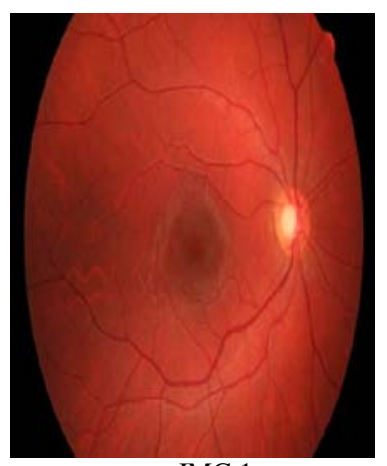

IMG 1

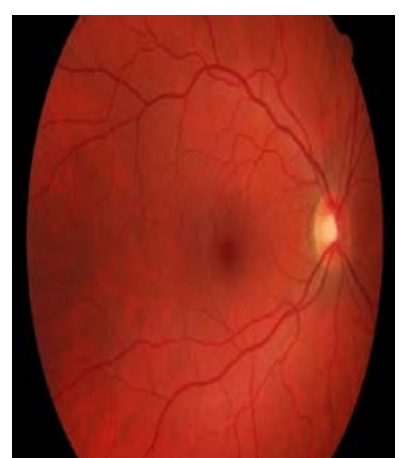

IMG 2

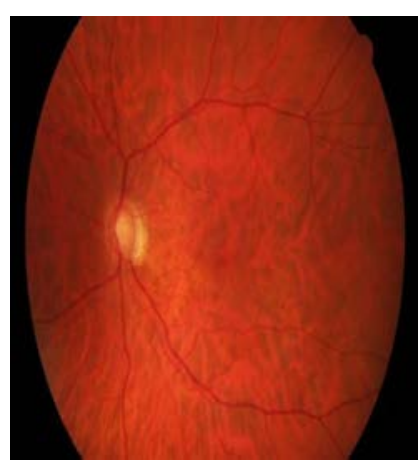

IMG 3

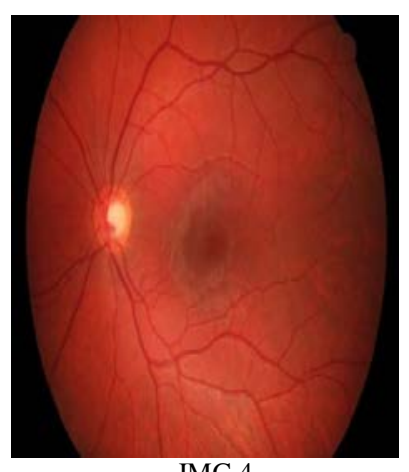

IMG 4

Fig. 2. Sample DR Images for Enhancement.

\section{IMPLEMENTATION AND RESULTS}

The proposed technique of medical image enhancement based on b-spline interpolation is implemented on a dataset of diabetic retinopathy. Special digital fundus cameras are used to obtain retinal images [19]. Diabetes mellitus has become a serious problem for health and study shows that it is the main source of deaths in diabetic's patients [11]. The quality of the fundus images is affected with various factors like, environmental conditions in which it is captured, brightness and angle of image, the lens of camera and the weather condition. Therefore, diabetic mellitus results are not good for segmentation and classification due to low brightness and contrast of captured images [19].

There are a few approaches already proposed to enhance the retinopathy images, like histogram equalization, Bezier curve, Hermite spline, etc. In this paper we made a comparison of our technique with a proposed technique named as diabetic retinopathy enhancement technique for fundus images [20]. To evaluate the sustainability of proposed techniques the experiments observed with histogram-oriented methods and statistical analysis [21]. The evaluation measures structure similarity index, entropy, peak signal to noise ratio and contrast to noise ratio are performed. The experiments are performed with MATLAB R2018a on core i5 64bit operating system.

\section{A. Histogram}

A histogram is the rectangular frequency of data items based on numerical form with equal size. It helps to perform statistical analysis. The dependent variables are along the vertical axis. While the independents are on horizontal axis. The result is shown in the form of a bar line in the graph [22]. The bar lines raised according to the data points given to them. In the given table the histogram of real and enhanced images is shown clearly.

The comparison of histograms of images showed that the frequencies of original images are high in starting points. On the other hand, the frequencies of enhanced images are medium in length and balanced in range [23]. The original and enhanced image histogram is shown in Fig. 2(c) and (d) respectively. It is cleared from the figure that the histogram equalization of enhanced images is balanced and return good results because the frequencies of images are distributed.

\section{B. Curve Lines}

A straight line in one direction is known as a line. The line that has more than one point and different direction is known as the curve line. The B-spline curve is important for contrast enhancement of images while the control points decide the movement of curve line [4]. The curve line of enhanced images is shown in Fig. 3(e). The original image has straight curve, the upward curve known as enhanced with brightness while the downward curve decreased the brightness of image.

\section{Performance Evaluation}

It's the statistical and mathematical analysis of enhancement technique to figure out the exact calculation of image. The difference of original image value and enhanced image value is known as enhancement. The evaluation is compared with the hybrid proposed fundus image enhancement using histogram equalization, contrast limited adaptive histogram equalization and exposure-based sub image histogram equalization (HE). The function of adaptive histogram equalization (AHE) is to compute different images histogram and reallocate intensities to enhance contrast of image [24]. Contrast limited adaptive histogram equalization (CLAHE) is good for low contrast images; it enhances its low contrast images using adjacent neighbor pixels to improve contrast. The contrast of the image is based on clip and slope of histogram height directly. If height is increased the contrasts also increase. It overcomes noise amplification as well [25]. Exposure based sub image histogram equalization (ESIHE) is used to enhance contrast and height of histogram. It gives the highest value of histogram height then already defined threshold values [26]. We performed comparison between HE, AHE, CLASHE, ESIHE techniques and proposed B-spline technique results. Please see quantitative results in Tables I, II, III and IV against each evaluation measure. 

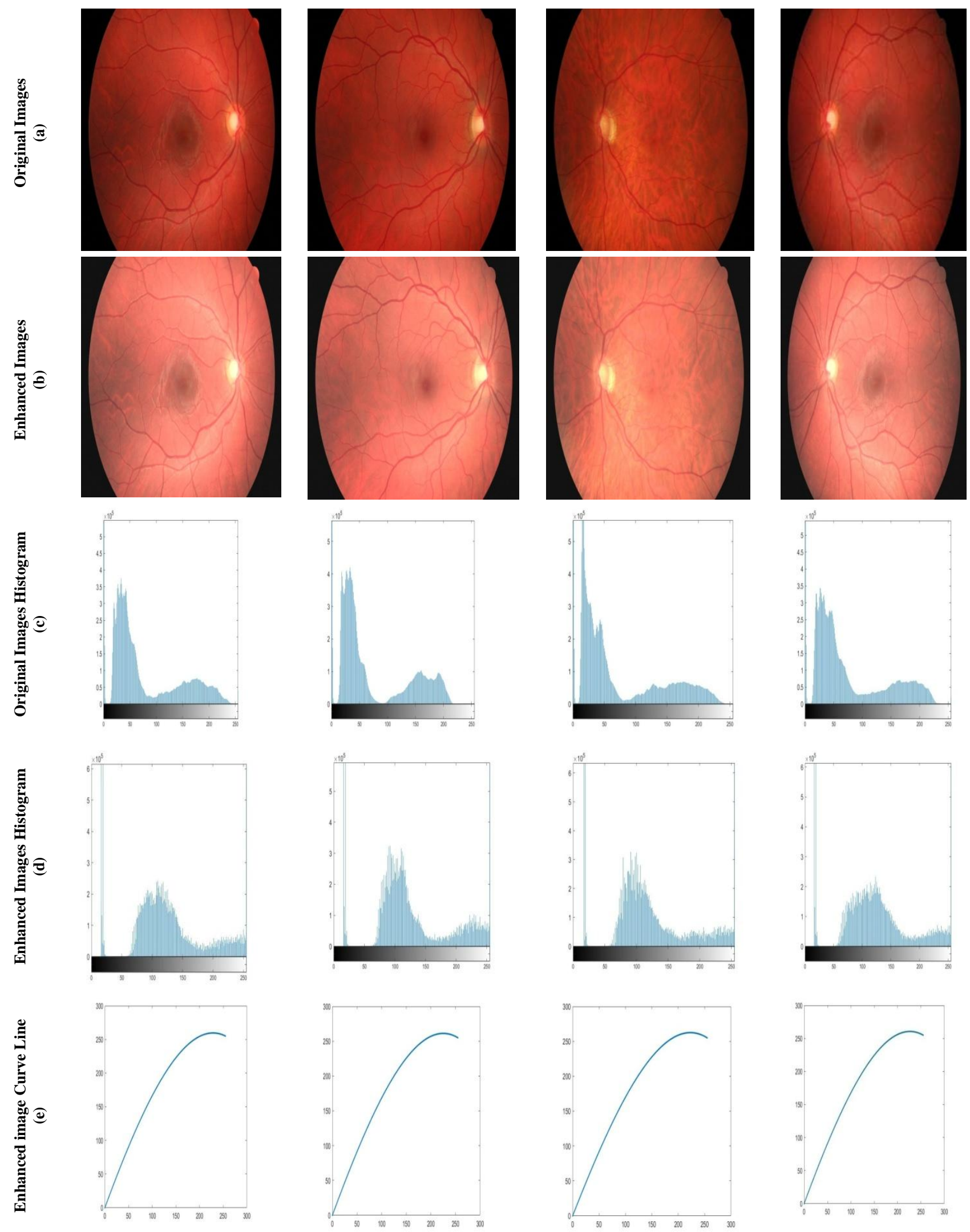

Fig. 3. (a) Sample DR Original Images (b) Enhanced Images (c) Hisgrogram of Orignial Images (d) Hisgrogram of Enhanced Iamges (e) Curve Line of Enhaced Iamges. 


\section{Evaluation Measures}

Following evaluation measures are used for quantitative evaluation of the proposed approach.

1) Structure Similarity Index Measure (SSIM): It is the measure that identifies the best results of original and enhanced images as described in equation (9).

$\operatorname{SSIM}(\mathrm{x}, \mathrm{y})=\frac{\left(2 \mu_{x} \mu_{y}+c_{1}\right)\left(2 \sigma_{x y}+c_{2}\right)}{\left(\mu_{x}^{2} \mu_{y}^{2}+c_{1}\right)\left(\sigma_{x}^{2} \sigma_{y}^{2}+c_{2}\right)}$

The maximum similarity index value is 1 . Image which has value near to 1 is called highest SSIM.

The SSIM value is enhanced if it is near to 1. After performing the experiments as shown in Table II, it is observed that the outcome value of HE, AHE and CLAHE not considerably significant to structure similarity index measure. But ESHIE nearly performed with enhancement of contrast in terms of SSIM. While the proposed technique is excellent to increase the contrast of an image. Structure similarity index of the dataset is starting from 0.9913 to 0.9917 . The contrast of image enhances the best level compared with other techniques.

2) Entropy: Entropy [12] provides the average of content information of an image. Its large value considers good results. But in some cases where loss of image information occurs it's not measured correctly [27].

$E(P)=\sum_{k-0}^{L-1} P_{k} \times \log P_{k}$

Entropy value of the given images is calculated as shown in Table III and found that the HE, AHE, EHIE values of entropy are less then original values. The CLAHE values are high with amplification of noise.

3) Peak Signal to Noise Ratio (PSNR): To calculate the worth of reconstructed image peak values of signal are identified. The more value of peak signals the stronger image was returned. Before measuring PSNR value the mean square error is identified.

$M S E=\frac{\sum_{i=1}^{M} \sum_{(j=1)}^{N}[X(i, j)-Y(i, j)]^{2}}{(M \times N)}$

$S N R=\frac{10 \log ^{10}(\text { peakvalue })^{2}}{M S E}$

The value of PSNR with all techniques is calculated in Table IV. The CLAHE is showing very low PSNR due to high noise ratio. The HE, AHE and ESHIE techniques provide enhancement of image contrast ratio. The proposed technique provides drastically changed results of PSNR because it's high contrast of image and low noise. The starting PSNR value is 60.24 to 60.45 on the chosen dataset. This result returns a very high contrast enhancement of images.

4) Contrast to Noise Ratio: To measure the value of noise and contrast in an output is known as the contrast to noise ratio. Now a days, it is widely used to measure medical images enhancement.
$C N R=\frac{\left|\mu_{1}-\mu_{2}\right|}{\sqrt{\sigma_{1}^{2}+\sigma_{2}^{2}}}$

CNR is another parameter to measure noise and contrast of image. The CLAHE provides the better result in histogram family that showing enhancement of images, proposed technique also returns good results on selected images. Table $\mathrm{V}$ shows the CNR comparison of different contrast techniques.

The enhancement returns better result of contrast. Results fluctuate according to the image CNR. The CNR of the image is changed from 6.64 to 13.09 . It means the standard deviation and expectation value of images are enhanced greatly.

TABLE II. COMPARISON USING EVALUATION MEASURE SSIM

\begin{tabular}{|l|l|l|l|l|l|}
\hline \multirow{5}{*}{ SSIM } & TECHNIQUES & IMG1 & IMG2 & IMG3 & IMG4 \\
\cline { 2 - 6 } & HE & 0.6705 & 0.6599 & 0.6356 & 0.6546 \\
\cline { 2 - 6 } & AHE & 0.7904 & 0.7832 & 0.8041 & 0.7888 \\
\cline { 2 - 6 } & CLAHE & 0.1498 & 0.1495 & 0.1497 & 0.1502 \\
\cline { 2 - 6 } & ESHIE & 0.9391 & 0.932 & 0.9367 & 0.9338 \\
\cline { 2 - 6 } & Proposed & $\mathbf{0 . 9 9 1 9}$ & $\mathbf{0 . 9 9 1 3}$ & $\mathbf{0 . 9 9 1 4}$ & $\mathbf{0 . 9 9 1 7}$ \\
\hline
\end{tabular}

TABLE III. COMPARISON USING EVALUATION MEASURE ENTROPY

\begin{tabular}{|l|l|l|l|l|l|}
\hline \multirow{5}{*}{ Entropy } & Techniques & IMG1 & IMG2 & IMG3 & IMG4 \\
\cline { 2 - 6 } & Original & 6.8979 & 6.6775 & 6.8096 & 6.9268 \\
\cline { 2 - 6 } & HE & 5.6178 & 5.6363 & 5.5438 & 5.6148 \\
\cline { 2 - 6 } & AHE & 6.7072 & 6.5338 & 6.5377 & 6.7421 \\
\cline { 2 - 6 } & CLAHE & 7.1793 & 7.0444 & 7.1434 & 7.1858 \\
\cline { 2 - 6 } & ESHIE & 6.7896 & 6.5811 & 6.7133 & 6.8135 \\
\cline { 2 - 6 } & Proposed & $\mathbf{6 . 6 8 3 1}$ & $\mathbf{6 . 6 4 0 0}$ & $\mathbf{6 . 5 9 0 0}$ & $\mathbf{6 . 7 3 0 0}$ \\
\hline
\end{tabular}

TABLE IV. COMPARISON USING EVALUATION MEASURE PSNR

\begin{tabular}{|l|l|l|l|l|l|}
\hline \multirow{5}{*}{ PSNR } & Techniques & IMG1 & IMG2 & IMG3 & IMG4 \\
\cline { 2 - 6 } & HE & 11.8856 & 11.3802 & 11.1596 & 11.6942 \\
\cline { 2 - 6 } & AHE & 21.3623 & 21.2975 & 21.8054 & 20.6336 \\
\cline { 2 - 6 } & CLAHE & 08.4919 & 08.8072 & 8.7615 & 08.7041 \\
\cline { 2 - 6 } & ESHIE & 28.0744 & 26.6361 & 26.9853 & 26.9576 \\
\cline { 2 - 6 } & Proposed & $\mathbf{6 0 . 4 5 0 0}$ & $\mathbf{6 0 . 2 4 0 0}$ & $\mathbf{6 0 . 2 5 0 0}$ & $\mathbf{6 0 . 3 8 0 0}$ \\
\hline
\end{tabular}

TABLE V. COMPARISON USING EVALUATION MEASURE CNR

\begin{tabular}{|l|l|l|l|l|l|}
\hline \multirow{5}{*}{ Techniques } & IMG1 & IMG2 & IMG3 & IMG4 \\
\cline { 2 - 6 } & Original & 12.6900 & 11.790 & 6.6400 & 15.7900 \\
\cline { 2 - 6 } & HE & 12.77600 & 11.8390 & 13.3737 & 15.8677 \\
\cline { 2 - 6 } & AHE & 17.3575 & 20.2954 & 17.7085 & 18.3116 \\
\cline { 2 - 6 } & CLAHE & 17.6047 & 22.5876 & 13.4085 & 20.1139 \\
\cline { 2 - 6 } & ESHIE & 12.0926 & 13.4324 & 14.2321 & 16.8950 \\
\cline { 2 - 6 } & Proposed & $\mathbf{1 2 . 8 9 0 0}$ & $\mathbf{1 2 . 0 1 0 0}$ & $\mathbf{1 3 . 0 9 0 0}$ & $\mathbf{1 7 . 4 0 0 0}$ \\
\hline
\end{tabular}




\section{CONCLUSION AND FUTURE WORK}

Enhancement of medical images is an important task while diagnosis of diseases using images. In this paper the diabetic based retinopathy images are enhanced by applying B-spline. There are many famous methods to enhance diabetic retinopathy images in the, but they are not robust with the results due to noise, loss of information. The proposed technique is used to enhance the contrast of the images automatically by using B-spline, which passes through every point of curve and provides the best and amplified enhancement of contrast. The B-spline curve is dynamic in nature and works like a Bezier curve and returns enhanced images without loss of any information. The curve is computed based on control points the resultant image luminance and contrast is then transformed according to the distance (D) measured by control points. Messidore and Drive dataset of fundus images are used to evaluate the proposed techniques. By performing experiments, the results are visually and statistically enhanced the contrast of the fundus images, while the noise is also removed. In future we will work on enhancement of CT scan and MRI images.

\section{REFERENCES}

[1] Y. Chang, C. Jung, P. Ke et al., "Automatic contrast-limited adaptive histogram equalization with dual gamma correction,” IEEE Access, vol. 6, pp. 11782-11792, 2018.

[2] K. Asghar, G. Gilanie, M. Saddique et al., "Automatic enhancement of digital images using cubic Bézier curve and Fourier transformation,” Malaysian Journal of Computer Science, vol. 30, no. 4, pp. 300-310, 2017.

[3] K. Akila, L. Jayashree, and A. Vasuki, "Mammographic image enhancement using indirect contrast enhancement techniques-a comparative study,” Procedia Computer Science, vol. 47, pp. 255-261, 2015.

[4] [4] C. Zhao, Z. Wang, H. Li et al., "A new approach for medical image enhancement based on luminance-level modulation and gradient modulation,” Biomedical Signal Processing and Control, vol. 48, pp. 189-196, 2019.

[5] R. Firoz, M. S. Ali, M. N. U. Khan et al., "Medical image enhancement using morphological transformation," Journal of Data Analysis and Information Processing, vol. 4, no. 01, pp. 1, 2016.

[6] Y.-P. Wang, Q. Wu, K. R. Castleman et al., "Chromosome image enhancement using multiscale differential operators,” IEEE transactions on medical imaging, vol. 22, no. 5, pp. 685-693, 2003.

[7] A. A. Wahab, M. I. M. Salim, J. Yunus et al., "Comparative Evaluation of Medical Thermal Image Enhancement Techniques for Breast Cancer Detection,” Journal of Engineering and Technological Sciences, vol. 50, no. 1, pp. 40-52, 2018.

[8] D.-Y. Tsai, and Y. Lee, "A method of medical image enhancement using wavelet-coefficient mapping functions." pp. 1091-1094.

[9] C. S. Tutika, C. Vallapaneni, B. KP et al., "Cubic spline interpolation segmenting over conventional segmentation procedures: application and advantages,” arXiv preprint arXiv:1803.04621, 2018.
[10] S. Dutta, B. C. Manideep, S. M. Basha et al., "Classification of diabetic retinopathy images by using deep learning models," International Journal of Grid and Distributed Computing, vol. 11, no. 1, pp. 89-106, 2018.

[11] T. Walter, J.-C. Klein, P. Massin et al., “A contribution of image processing to the diagnosis of diabetic retinopathy-detection of exudates in color fundus images of the human retina," IEEE transactions on medical imaging, vol. 21, no. 10, pp. 1236-1243, 2002.

[12] M. D. Abràmoff, Y. Lou, A. Erginay et al., "Improved automated detection of diabetic retinopathy on a publicly available dataset through integration of deep learning," Investigative ophthalmology \& visual science, vol. 57, no. 13, pp. 5200-5206, 2016.

[13] T. Blu, and M. Unser, "The fractional spline wavelet transform: definition end implementation." pp. 512-515.

[14] S. U. Talha, T. Mairaj, W. Khan et al., "Interpolation based enhancement of sparse-view projection data for low dose FBP reconstruction." pp. 1-6.

[15] N. Al-Najdawi, M. Biltawi, and S. Tedmori, "Mammogram image visual enhancement, mass segmentation and classification," Applied Soft Computing, vol. 35, pp. 175-185, 2015.

[16] T. M. Lehmann, C. Gonner, and K. Spitzer, “Addendum: B-spline interpolation in medical image processing," IEEE transactions on medical imaging, vol. 20, no. 7, pp. 660-665, 2001.

[17] X. Fu, D. Zeng, Y. Huang et al., "A fusion-based enhancing method for weakly illuminated images,” Signal Processing, vol. 129, pp. 82-96, 2016.

[18] E. Decencière, X. Zhang, G. Cazuguel et al., "Feedback on a publicly distributed image database: the Messidor database,” Image Analysis \& Stereology, vol. 33, no. 3, pp. 231-234, 2014.

[19] J. I. Orlando, E. Prokofyeva, M. del Fresno et al., “An ensemble deep learning-based approach for red lesion detection in fundus images," Computer methods and programs in biomedicine, vol. 153, pp. 115-127, 2018.

[20] I. Qureshi, J. Ma, and Q. Abbas, "Recent Development on Detection Methods for the Diagnosis of Diabetic Retinopathy,” Symmetry, vol. 11, no. 6, pp. 749, 2019.

[21] K.-K. Maninis, J. Pont-Tuset, P. Arbeláez et al., "Deep retinal image understanding." pp. 140-148.

[22] S.-W. Lee, and J. K. Paik, "Image interpolation using adaptive fast Bspline filtering." pp. 177-180.

[23] C. Y. Wong, G. Jiang, M. A. Rahman et al., "Histogram equalization and optimal profile compression-based approach for colour image enhancement," Journal of Visual Communication and Image Representation, vol. 38, pp. 802-813, 2016.

[24] O. Patel, Y. P. Maravi, and S. Sharma, "A comparative study of histogram equalization based image enhancement techniques for brightness preservation and contrast enhancement," arXiv preprint arXiv:1311.4033, 2013.

[25] B. Singh, and S. Patel, "Efficient medical image enhancement using CLAHE enhancement and wavelet fusion," International Journal of Computer Applications, vol. 167, no. 5, pp. 0975-8887, 2017.

[26] I. Qureshi, J. Ma, and K. Shaheed, “A hybrid proposed fundus image enhancement framework for diabetic retinopathy,” Algorithms, vol. 12, no. 1, pp. 14, 2019.

[27] A. Karci, "Fractional order entropy: New perspectives,” Optik, vol. 127, no. 20, pp. 9172-9177, 2016 